\section{(2) OPEN ACCESS}

\title{
Mediterranean diet intervention in overweight and obese subjects lowers plasma cholesterol and causes changes in the gut microbiome and metabolome independently of energy intake
}

\author{
Victoria Meslier, ${ }^{1}$ Manolo Laiola, ${ }^{2}$ Henrik Munch Roager (D) , ${ }^{3}$ Francesca De Filippis, ${ }^{2,4}$ \\ Hugo Roume, ${ }^{1}$ Benoit Quinquis, ${ }^{1}$ Rosalba Giacco, ${ }^{5}$ Ilario Mennella, ${ }^{2}$ Rosalia Ferracane, ${ }^{2}$ \\ Nicolas Pons, ${ }^{1}$ Edoardo Pasolli, ${ }^{2,4}$ Angela Rivellese, ${ }^{4,6}$ Lars Ove Dragsted (1) , ${ }^{3}$ \\ Paola Vitaglione, ${ }^{2,4}$ Stanislav Dusko Ehrlich (D) , ${ }^{1}$ Danilo Ercolini (D) ${ }^{2,4}$
}

\begin{abstract}
- Additional material is published online only. To view please visit the journal online (http://dx.doi.org/10.1136/ gutjnl-2019-320438).

For numbered affiliations see end of article.
\end{abstract}

\section{Correspondence to}

Professor Danilo Ercolini and Dr Paola Vitaglione, Department of Agricultural Sciences, University of Naples Federico II, Portici 80055, Italy;

ercolini@unina.it, paola. vitaglione@unina.it and Professor Stanislav Dusko Ehrlich, Metagenopolis, INRAE, 78350 Jouy en Josas, France; stanislav.ehrlich@inra.fr

VM, ML and HMR contributed equally.

Received 10 December 2019 Revised 17 January 2020 Accepted 21 January 2020 Published Online First 19 February 2020

\section{Linked}

http://dx.doi.org/10.1136/ gutjnl-2019-319654 - http://dx.doi.org/10.1136/ gutjnl-2020-320781

\section{Check for updates}

(c) Author(s) (or their employer(s)) 2020. Re-use permitted under CC BY-NC. No commercial re-use. See rights and permissions. Published by BMJ.

To cite: Meslier V, Laiola M, Roager HM, et al. Gut

2020:69:1258-1268.

\section{ABSTRACT \\ Objectives This study aimed to explore the effects} of an isocaloric Mediterranean diet (MD) intervention on metabolic health, gut microbiome and systemic metabolome in subjects with lifestyle risk factors for metabolic disease.

Design Eighty-two healthy overweight and obese subjects with a habitually low intake of fruit and vegetables and a sedentary lifestyle participated in a parallel 8-week randomised controlled trial. Forty-three participants consumed an MD tailored to their habitual energy intakes (MedD), and 39 maintained their regular diets (ConD). Dietary adherence, metabolic parameters, gut microbiome and systemic metabolome were monitored over the study period.

Results Increased MD adherence in the MedD group successfully reprogrammed subjects' intake of fibre and animal proteins. Compliance was confirmed by lowered levels of carnitine in plasma and urine. Significant reductions in plasma cholesterol (primary outcome) and faecal bile acids occurred in the MedD compared with the ConD group. Shotgun metagenomics showed gut microbiome changes that reflected individual MD adherence and increase in gene richness in participants who reduced systemic inflammation over the intervention. The MD intervention led to increased levels of the fibre-degrading Faecalibacterium prausnitzii and of genes for microbial carbohydrate degradation linked to butyrate metabolism. The dietary changes in the MedD group led to increased urinary urolithins, faecal bile acid degradation and insulin sensitivity that co-varied with specific microbial taxa.

Conclusion Switching subjects to an MD while maintaining their energy intake reduced their blood cholesterol and caused multiple changes in their microbiome and metabolome that are relevant in future strategies for the improvement of metabolic health.

\section{INTRODUCTION}

Diet is a fundamental factor affecting gut health. Mounting evidence highlights that diets richer in plant-based rather than animal-based foods could represent healthier choices to prevent disease. ${ }^{12}$

\section{Significance of this study}

What is already known about this subject?

- Diet fundamentally influences gut health.

- Mediterranean diet (MD) is a recommended nutritional pattern with known beneficial effects including the prevention of several types of disease.

- Current knowledge of the effect of diet on microbiome-mediated health outcomes in humans relies mainly on observational studies.

What are the new findings?

- An isocaloric 8-week intervention with an MD in obese and overweight subjects leads to a decrease in total, low-density lipoprotein and high-density lipoprotein plasma cholesterol and faecal bile acids levels independently of energy intake, the decrease in cholesterol being proportional to MD adherence rates.

- The MD intervention causes microbiome changes with increased gene richness in individuals with reduced inflammation, a rise in the fibre-degrading Faecalibacterium prausnitzii accompanied by a decrease of the potentially proinflammatory Ruminococcus gnavus.

- Increased MD adherence generates consistent metabolome changes, with lower plasma and urinary carnitine levels and protein degradation products concomitant with the increase of dietary biomarkers of plant-based foods.

- The consumption of plant foods typical of an MD can determine increase in urolithin production, decreased insulin resistance and bile acid levels, and such changes are all consistently related to baseline levels and variations of the microbial species involved in these specific metabolic features.

The Mediterranean diet (MD) is a recommended nutritional pattern with evidence of beneficial effects including the prevention of several types of disease, such as cardiovascular disease (CVD), type 
Significance of this study

\section{How might it impact on clinical practice in the foreseeable future?}

- The results of this study clearly show that a change in dietary behaviour without any concomitant change in individual energy intake, macronutrient intake and physical activity can lower blood cholesterol, already after 4 weeks, in a population with cardiometabolic risk for unhealthy lifestyle.

- The beneficial dietary changes are in line with an increased adherence to MD and include a reduction of intake of meat and refined cereal products and increased intakes of fruit, vegetables, wholegrain cereal products, legumes and fish, along with a daily consumption of nuts.

- Some individuals harbour a gut microbiome that is more susceptible to MD-induced changes and experience further clinical advantages such as amelioration of insulin sensitivity and of inflammatory status.

2 diabetes, obesity, inflammatory diseases, degenerative diseases and cancer. ${ }^{3-5}$

The microbiome partly but significantly affects individual metabolism and how one responds to changes in dietary habits. ${ }^{67}$ Host health is influenced by microbiome composition and by microbial metabolites that can be produced from host metabolic intermediates or from dietary precursors. ${ }^{8}$ Therefore, current trends in personalised nutrition suggest that diet can be used to modulate microbiome composition and function. ${ }^{9}{ }^{10}$ Indeed, the production of beneficial microbial metabolites can be increased, and the production of detrimental metabolites can be reduced by modulating nutrient intake and supplying a beneficial pattern of key precursors to the microbiome.

The current knowledge of the role of diet on microbiomemediated health outcomes in humans mainly relies on observational studies in which confounding factors affect the conclusions. ${ }^{11}$ Intervention studies to address the causal effects of diet on microbiome functions are still scarce or have been performed in animal models, ${ }^{12}$ and this lack of knowledge also applies to the MD. ${ }^{13}$ Despite their cost and labour-intensiveness, randomised controlled trials (RCTs) are the gold standard for evidence-based medicine and are an appropriate tool for identifying a causal relationship of a specific nutrient or diet on a health outcome in humans. ${ }^{14} 15$

A Western diet is characterised by an excessive intake of foods with a high energy density and that are rich in fats, sugars and animal proteins, as well as a very low intake of fruits and vegetables. Such a dietary style, accompanied by low levels of physical activity, promotes inflammation ${ }^{16}$ and predisposes individuals to obesity, CVD, type 2 diabetes and metabolic syndrome. ${ }^{17} 18$ Because obesity is highly prevalent worldwide and is recognised as an independent risk factor for metabolic-driven chronic diseases, efforts need to be made urgently to provide evidencebased recommendations for healthy dietary patterns.

The aim of this study was to evaluate the effect of an individually tailored MD intervention in subjects at increased risk of cardiovascular disease.

\section{MATERIALS AND METHODS}

\section{Study design and population}

We investigated the gut microbiome, faecal, blood and urinary metabolomic profiles in 82 overweight/obese subjects in response to an 8-week isocaloric dietary intervention with an MD or a control diet. Each participant provided written informed consent and received no financial compensation. The trial was registered at ClinicalTrials.gov (number NCT03071718). The protocol ended when the last group of participants completed the protocol (Study Start Date: June 2016; Actual Primary Completion Date: July 2017; Actual Study Completion Date: February 2019).

The study design, selection criteria and participant flow throughout the study are reported in the online supplementary materials (online supplementary figure 1). Plasma lipids (including plasma cholesterol and triglycerides) and faecal levels of short-chain fatty acids (SCFAs) were registered as primary outcomes of the study, while changes in gut microbiota and some intermediate markers of metabolic disease, such as blood pressure, fasting blood glucose, serum high sensitivity $\mathrm{C}$ reactive protein (hs-CRP), urinary and plasma trimethylamine oxide (TMAO), plasma gastrointestinal peptides and urinary polyphenols, were secondary outcomes (detection methods described in the online supplementary material). Briefly, 334 potentially eligible adults were screened on the basis of the inclusion/exclusion criteria, including medical and lifestyle conditions (ie, habitual diet and physical activity) (detailed criteria are in the online supplementary material). Adherence to the MD was estimated by using the 11-unit dietary score and is reported as the Italian Mediterranean Index (MD index). ${ }^{19}$

Eighty-two subjects (43 female and 39 male, average body mass index (BMI) $31.1 \pm 4.5 \mathrm{~kg} / \mathrm{m}^{2}$, age $43 \pm 12$ years, further baseline features in the online supplementary table 1) were selected, enrolled and randomised between the two intervention arms of the parallel study design, that is, MedD or ConD.

\section{Dietary intervention}

Each participant in the MedD group consumed an individually tailored diet that maintained the daily energy and macronutrient intake of the habitual diet and guaranteed a dietary pattern typical of the MD. Participants in the ConD group were asked to maintain their habitual diet. Individual compliance with the protocol was assessed every 2 weeks by self-recorded 7-day food diaries and physical activity questionnaires. Visits and sample collection were performed at baseline, 4 weeks and 8 weeks (full details reported in the online supplementary material).

\section{Metabolomics}

Untargeted urine, serum and faeces metabolomics, ${ }^{20}{ }^{21}$ as well as targeted quantification of bile acids (BAs) and SCFAs in the faeces, ${ }^{22} 23$ were performed by ultra-high-performance liquid chromatography mass spectrometry. Trimethylamine N-oxide (TMAO), carnitine, choline, creatinine, betaine in plasma and urine, as well as urinary urolithins, were also determined by targeted metabolomics using liquid chromatography tandem mass spectrometry. Details are available in the online supplementary material and online supplementary tables $2-3$.

\section{Metagenomics}

A full description of the sampling, sequencing and data analysis procedures is reported in the online supplementary material. DNA libraries were sequenced using the Ion Proton Sequencer (ThermoFisher Scientific, Waltham, USA), with a minimum of 20 million $150 \mathrm{bp}$ high-quality reads generated per library. Metagenomic species pangenome (MSP) ${ }^{24}$ was used to identify and quantify species associated with the 9.9-million-gene integrated reference catalogue. The functional potentials of the intestinal gut microbiota were determined by using the in-house 
FAnToMet pipeline as described in the online supplementary material.

\section{Statistical analysis}

Statistical analysis and visualisation were carried out in $\mathrm{R}$ environment V.3.4.2 (https://www.r-project.org). ggpubr and $P M C M R \mathrm{R}$ packages were used to assess significant differences. Variations in dietary and clinical variables at specific time points compared with baseline values between the MedD and ConD groups were evaluated by two-way analysis of variance with repeated measures and Tukey's post hoc test. Non-parametric Wilcoxon signed-rank test (testRelations function of momr R package) was performed to compare means between ConD and MedD subjects at each time point, while the post hoc Nemenyi test for multiple comparisons following the Friedman test was used within each group.

Pairwise Spearman's rank correlations were used to estimate the overall similarity of the microbiome and metabolome within the MedD and ConD groups and between time points (baseline vs 4 weeks and 4 weeks vs 8 weeks). The same test was applied to the microbiome, dietary variables, clinical markers and targeted metabolome datasets. Adjustments were performed using the Benjamini-Hochberg procedure. Correlations were visualised using the ComplexHeatmap package. ${ }^{25}$

Machine learning-based classification ${ }^{26}$ of metabolomics data and further details on data analysis and visualisation are provided in the online supplementary material.

\section{Patient and public involvement}

Patient and public involvement was not considered in this research.

\section{Data availability}

Metagenomic reads generated in this study are available (without conditions of reuse) under the accession number PRJEB33500 at the European Nucleotide Archive (ENA) in EBI (https://www. ebi.ac.uk/ena/data/view/PRJEB33500).

\section{RESULTS}

MD lowered plasma cholesterol in the overall population

No significant differences in anthropometric measures or clinical variables monitored in blood and urine samples were observed between the ConD $(n=39)$ and MedD $(n=43)$ groups at baseline (online supplementary table 1 ). Regarding the primary outcomes, as a consequence of the intervention, the participants in the MedD showed a significant decrease in total plasma cholesterol (figure 1) and high-density lipoprotein (HDL)-cholesterol after 4 weeks compared with the ConD group (online supplementary table 1). No changes in any of the secondary outcomes such as blood glucose, serum hs-CRP, plasma insulin, TMAO or any intermediate markers of metabolic disease (glucagon, ghrelin, GIP, GLP-1, leptin, C-peptide, resistin, visfatin and PAI-1) were observed (online supplementary table 1).

Successful compliance to the protocol and cholesterol decrease proportional to MD adherence rates

Adherence to the MD significantly increased in the MedD group at 4 and 8 weeks compared with the baseline (figure 1A) and was highly correlated with the Healthy Food Diversity (HFD) index ${ }^{27}$ (online supplementary figure 2). Significant percentage changes in dietary and metabolic variables are shown in figure 1B. Participants in the MedD group significantly increased their daily intake of dietary fibre by twofold and their dietary vegetable:animal protein ratio by 2.5 -fold over the intervention compared with the ConD group $(\mathrm{p}<0.001$, figure $1 \mathrm{C}, \mathrm{D})$. A significant reduction in saturated fat intake and an increase in polyunsaturated fat intake was also achieved $(\mathrm{p}<0.001$, figure 1E). These changes in nutrient intake in the MedD versus the ConD group were due to increased consumption of fruits, vegetables, nuts, wholegrain cereals and fish products concurrent with reduced consumption of refined cereals, dairy and meat products. The reduced consumption of meat products was confirmed by the reduction of the dietary intake biomarker of these foods in the MedD versus ConD group, that is, the concentration of carnitine in the plasma $(14 \%$ and $11 \%$ reductions after 4 and 8 weeks,

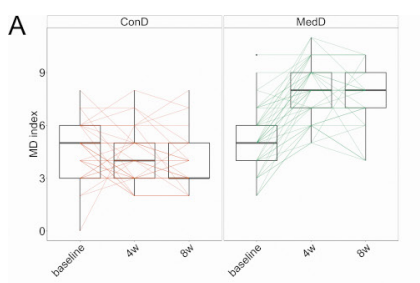

B
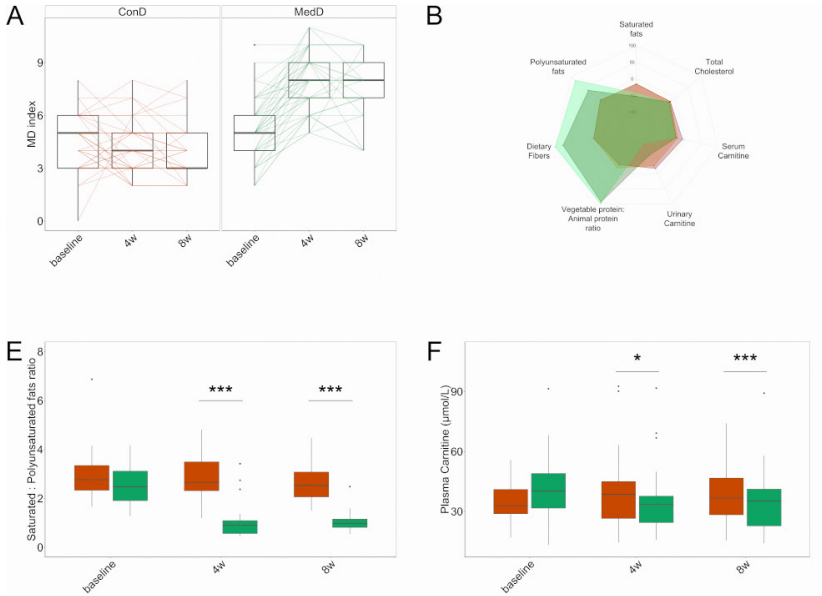
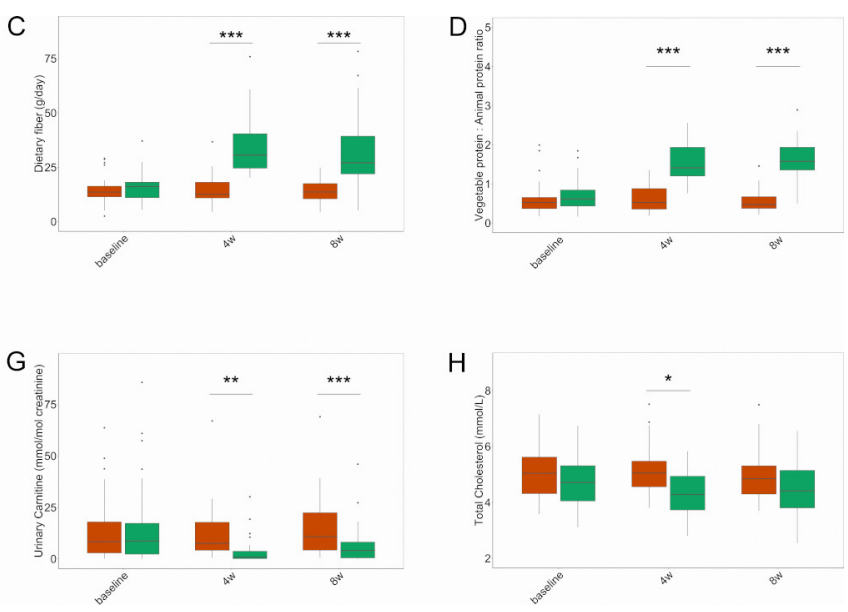

Figure 1 Adherence to the Mediterranean diet (MD) and changes in dietary and metabolic variables. (A) Box plots showing MD index score for controls (ConD) or treated subjects (MedD) during the intervention, the significance was tested by applying the post hoc Friedman-Nemenyi test for pairwise test of multiple comparisons within each group. (B) Percentage changes in dietary and metabolic variables are represented as spider chart. Changes in levels of dietary components consumption including (C) dietary fibre, (D) vegetable proteins/animal proteins ratio, (E) saturated to polyunsaturated fats ratio. Reduction in serum and urinary markers such as (F) plasma carnitine, $(\mathrm{G})$ urinary carnitine and $(\mathrm{H})$ total cholesterol. The significance was tested by applying unpaired Wilcoxon rank-sum tests for variation at the specific time point compared with baseline in MedD versus ConD. Orange boxes refer to controls and green boxes to Mediterranean subjects, respectively. Baseline, 0 weeks; $4 \mathrm{w}$, 4 weeks; 8 w, 8 weeks of nutritional intervention $\left({ }^{*} p<0.05,{ }^{* *} p<0.01\right.$ and $\left.{ }^{* * *} p<0.001\right)$. 
$\mathrm{p}<0.05$ and $\mathrm{p}<0.001$, respectively, figure $1 \mathrm{~F})$ and urine $(75 \%$ and $51 \%$ reductions after 4 and 8 weeks, $p<0.01$ and $p<0.001$, respectively, figure $1 \mathrm{G}$ ).

During the run-in period, some subjects (12 in the MedD group and 6 in the ConD group) undesirably increased their intake of fruit and vegetables above three servings/day compared with their consumption at the time of enrolment. We decided to strictly focus on the population who maintained a baseline dietary intake of fruits and vegetables $<3$ servings/day. In addition, two subjects were not considered because not all the faecal samples were available. Therefore, the subsequent data analyses were carried out with a subgroup of 62 subjects, 32 in the ConD group and 30 in the MedD group. High compliance with the intervention was confirmed in both groups. Changes in dietary intake of nutrients from several food categories are shown in online supplementary figure 3 . The effect of MedD on plasma cholesterol was confirmed in this subgroup. Indeed, following 4 weeks of intervention with an MD, a significant reduction $(\mathrm{p}=0.03)$ in plasma LDL-cholesterol from $2.90 \pm 0.13 \mathrm{mmol} / \mathrm{L}$ at baseline to $2.66 \pm 0.12 \mathrm{mmol} / \mathrm{L}$ at 4 weeks was observed in the MedD group compared with the change in the ConD group (3.24 $\pm 0.13 \mathrm{mmol} / \mathrm{L}$ at baseline to $3.25 \pm 0.12 \mathrm{mmol} / \mathrm{L}$ at 4 weeks), and a significant reduction $(\mathrm{p}=0.02)$ in plasma HDL-cholesterol from $1.26 \pm 0.05 \mathrm{mmol} / \mathrm{L}$ at baseline to $1.18 \pm 0.04 \mathrm{mmol} / \mathrm{L}$ at 4 weeks was observed in the MedD group compared with the change in the ConD group $(1.21 \pm 0.05 \mathrm{mmol} / \mathrm{L}$ at baseline to $1.25 \pm 0.05 \mathrm{mmol} / \mathrm{L}$ at 4 weeks).

Interestingly, decrease in cholesterol levels was proportional to MD adherence rate. By applying a linear model, it was found that each unit increase in the MD index corresponded to $\simeq 2 \%$ reduction in total plasma cholesterol $(p=0.003$, online supplementary figure 4$)$, a $2 \%$ reduction in plasma LDL-cholesterol $(\mathrm{p}=0.01)$ and $1 \%$ reduction in plasma HDL-cholesterol $(p=0.04)$ after adjustment for age, sex, BMI and energy intake.
Diet-induced metabolome changes with release of biomarkers of MD consumption

We measured approximately 11000 molecular features in all our participants during the intervention (2200 in faeces, 4125 in blood and 4645 in urine). A list of annotated metabolites is provided in online supplementary table 4 and the evidence substantiating the annotation of diet-responsive metabolites is provided in online supplementary material (online supplementary figures 5-25). Clear shifts in the metabolomic profiles were observed in the MedD group after the intervention compared with the baseline conditions and the ConD group (figure 2). Decreasing Spearman's correlation coefficients (4 weeks vs baseline; 8 weeks vs baseline) indicated a significant change in the urine metabolic profiles after $4(p=0.01)$ and 8 weeks $(\mathrm{p}=0.01)$ of intervention in the MedD group versus the ConD group. In order to validate the robustness of the shifts observed, we used a machine learning-based classification approach ${ }^{26}$ (area under the curve (AUC) $=0.88$ and 0.87 between the ConD and MedD groups at 4 and 8 weeks, respectively; as a control, $\mathrm{AUC}=0.52$ was observed at baseline), which supported the metabolome changes found. In agreement with the replacement of refined cereal with wholegrain products and the replacement of meat, eggs and dairy products with fishery products, legumes and provided nuts, we found increased levels of the biomarkers of wholegrains (3-(3,5-dihydroxyphenyl) propanoic acid-glucuronide), ${ }^{28}$ legumes (tryptophan betaine), ${ }^{29}$ vegetables/berries (oxindole- 3 -acetic acid) ${ }^{30}$ and nuts (urolithins) ${ }^{31}$ in the MedD group, while biomarkers of meat (carnitine), ${ }^{32} \mathrm{BAs}$, leucine and isoleucine were more closely linked to the ConD group (figure 2). Notably, no change in urine or serum TMAO was observed, possibly due to contrasting effects of increasing intake of fish and lowering meat-derived proteins in the MedD group.
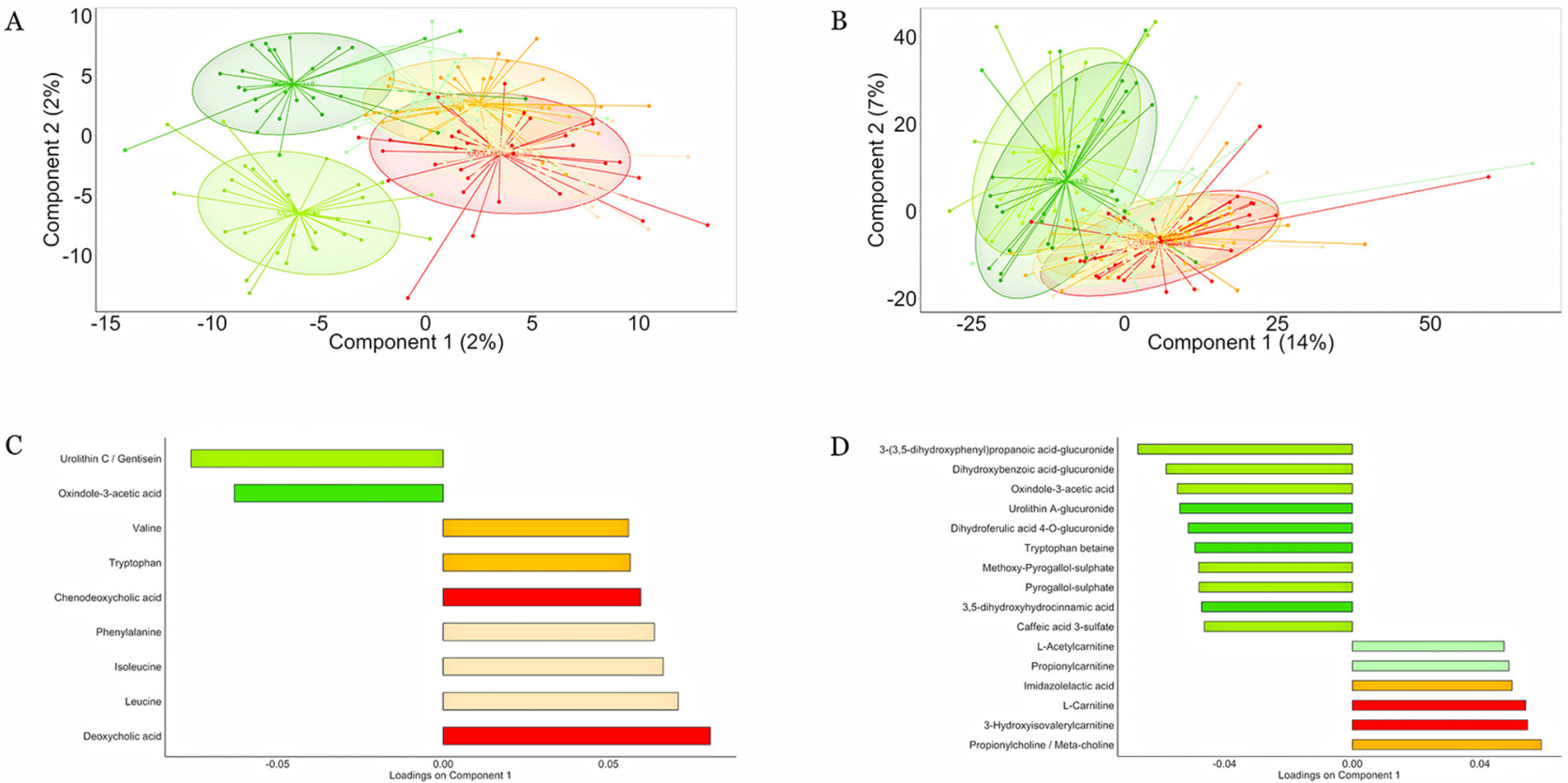

Figure 2 Mediterranean diet changes the intestinal and systemic metabolome. Partial least squares discriminant analysis plots based on molecular features detected in (A) faeces and (B) urine. Subjects belonging to different categories were coloured according to diet and time points: MedD subjects at baseline (light green), after 4 weeks (green) and 8 weeks of intervention (dark green). ConD subjects at baseline (light orange), after 4 weeks (orange) and 8 weeks (dark orange) of intervention. The loading plots display vectors that contributed the most to variability of individual dataset; variables explaining the variance between the groups in (C) faecal and (D) urine metabolome are reported as bar plots. 

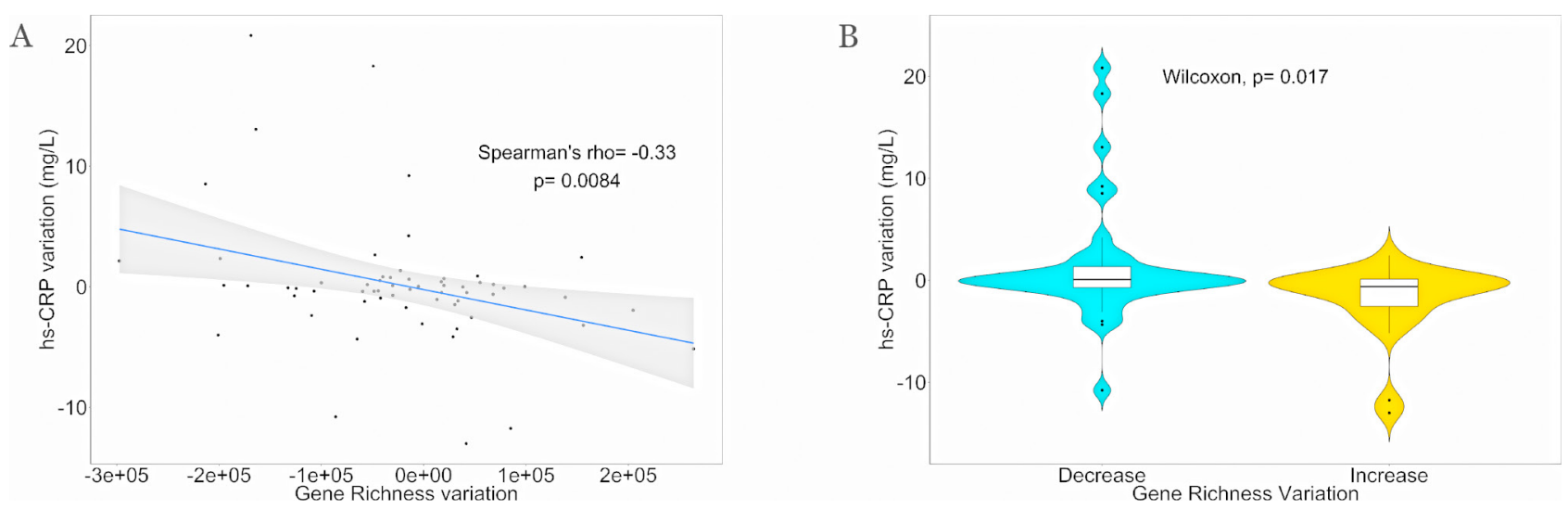

Figure 3 Microbial diversity richness anticorrelates with inflammation. (A) Spearman's correlation between variation of gut microbial gene richness and individual inflammatory status (serum hs-CRP) variation at the end of trial; $n$ observation=62. (B) Violin plot showing differences in serum hs-CRP variation between subjects increasing ( $n=25$, yellow) compared with subjects decreasing $(n=37$, light blue) gene richness at the end of trial. Statistical differences between groups were determined using unpaired Wilcoxon rank-sum tests. hs-CRP, high sensitivity $\mathrm{C}$ reactive protein.

MD-mediated increase in biomarkers of wholegrain (benzoxazinoids, pipecolic acid betaine), vegetable (oxindole-3-acetic acid), legume (tryptophan betaine, pyrogallol-sulfate), nuts (urolithins) and fish (3-carboxy-4-methyl-5-propyl-2-furanprop ionic acid) consumption and decrease in meat (carnitine) and protein degradation products such as branched-chain amino acids (BCAAs), aromatic amino acids, $N$-acetylcadaverine and microbial-derived proteolysis products ( $p$-cresol sulfate, indoxyl sulfate, phenylacetylglutamine) was further confirmed by their significant links with the MD index (online supplementary figure 26). Finally, a range of host-derived short-chain and mediumchain acylcarnitines was significantly reduced in the urine following the MD intervention, indicating a shift in substrates for energy metabolism from fat to complex carbohydrates and protein. ${ }^{33}$

\section{Gut microbiome composition is modulated by adherence to the MD}

Gene (average $=5551310.5 \pm 120191$ ) and MSP richness (average $=230.9 \pm 53.1$ ) metrics were maintained during the intervention. However, a significant inverse correlation was found between the variation in gut microbial gene richness and individual inflammatory status evaluated by serum hs-CRP variations (figure $3 \mathrm{~A})$. Subjects showing increased gene richness displayed significantly lower levels of serum hs-CRP after 8 weeks of the dietary intervention (figure 3B).

The increased adherence to the MD in the first 4 weeks corresponded to a decrease in the microbiome similarity in the MedD group during the same time interval, suggesting a MD-induced rearrangement of the gut microbiome composition (figure 4). This change was not observed either in the ConD group over the entire intervention or in the MedD group between 4 weeks and 8 weeks, that is, in intervention conditions when participants did not change their adherence to the MD.

While a negligible number of differentially abundant MSPs was found at baseline between the ConD and MedD groups $(n=27$ MSPs, online supplementary figure 27 and online supplementary table 5), more contrasting species were observed at $4(n=77$ MSPs) and 8 weeks ( $n=44$ MSPs, online supplementary figures 28 and 29 , online supplementary table 5), with the proportion of contrasting species consistently linked to the MD adherence evaluated by MD index (online supplementary tables 5 and 6).

During the increasing MD adherence phase (baseline-4 weeks), Ruthenibacterium lactatiformans, Flavonifractor plautii,
Parabacteroides merdae, Ruminococcus torques and Ruminococcus gnavus were significantly reduced in the MedD compared with the ConD group, along with Streptococcus thermophilus, a well-known marker of dairy product consumption. In contrast, five members of the Faecalibacterium prausnitzii clade were enriched in the MedD compared with the ConD group at either 4 or 8 weeks (online supplementary tables 5 and 6), along with several members of the Roseburia and Lachnospiraceae taxa. Consistently, MSPs enriched in the MedD group after 4 weeks were significantly linked to MD food biomarkers (online supplementary figure 30 ).

While only five gut metabolic modules (GMMs) were different between the diets (4\% of functional potential variation; online supplementary table 7 ) at baseline, $18 \%$ variation in the metabolic potential captured by GMM was observed after 4 weeks. Several GMMs $(n=19)$ were enriched in the MedD group, mainly including pathways related to amino acid and carbohydrate degradation. The pathways also included triglyceride and glycoprotein degradation and conversion of acetyl-CoA and glutamate degradation, both leading to crotonyl-CoA, a possible precursor of butyrate metabolism (online supplementary table 7). Although only $6 \%$ variation was observed after 8 weeks, enrichment in glutamate degradation to crotonyl-CoA was maintained in the MedD group. This pathway was significantly linked to the levels of F. prausnitzii msp_0388 (Spearman's rho $=0.73$, $\mathrm{p}<10 \mathrm{e}-6$, online supplementary figure 30 ).

Altogether, by integrating the three meta-omics datasets, ${ }^{34}$ we observed a separation of the ConD and MedD groups on the basis of microbiome diversity, functional modules and metabolomic profiles (Hotelling $\mathrm{T}^{2}=40.95, \mathrm{p}<7.038 \mathrm{e}-12$; online supplementary figure 31 ) corroborating the changes induced by the MD intervention.

\section{MD intervention affects microbiome functions}

We measured a number of metabolites associated with gut microbial metabolism to investigate the effect of the MD dietary intervention on health-related microbial activities. Urinary levels of urolithin glucuronides increased in the MedD compared with the ConD group (table 1). Such increase was consistently linked with the levels of urolithin producers in the microbiome, including, among others, members of the Eggerthellaceae family (online supplementary table 8), and with the consumption of nuts that were the sole dietary source of ellagitannins significantly increased in the MedD group. Interestingly, urolithin production 

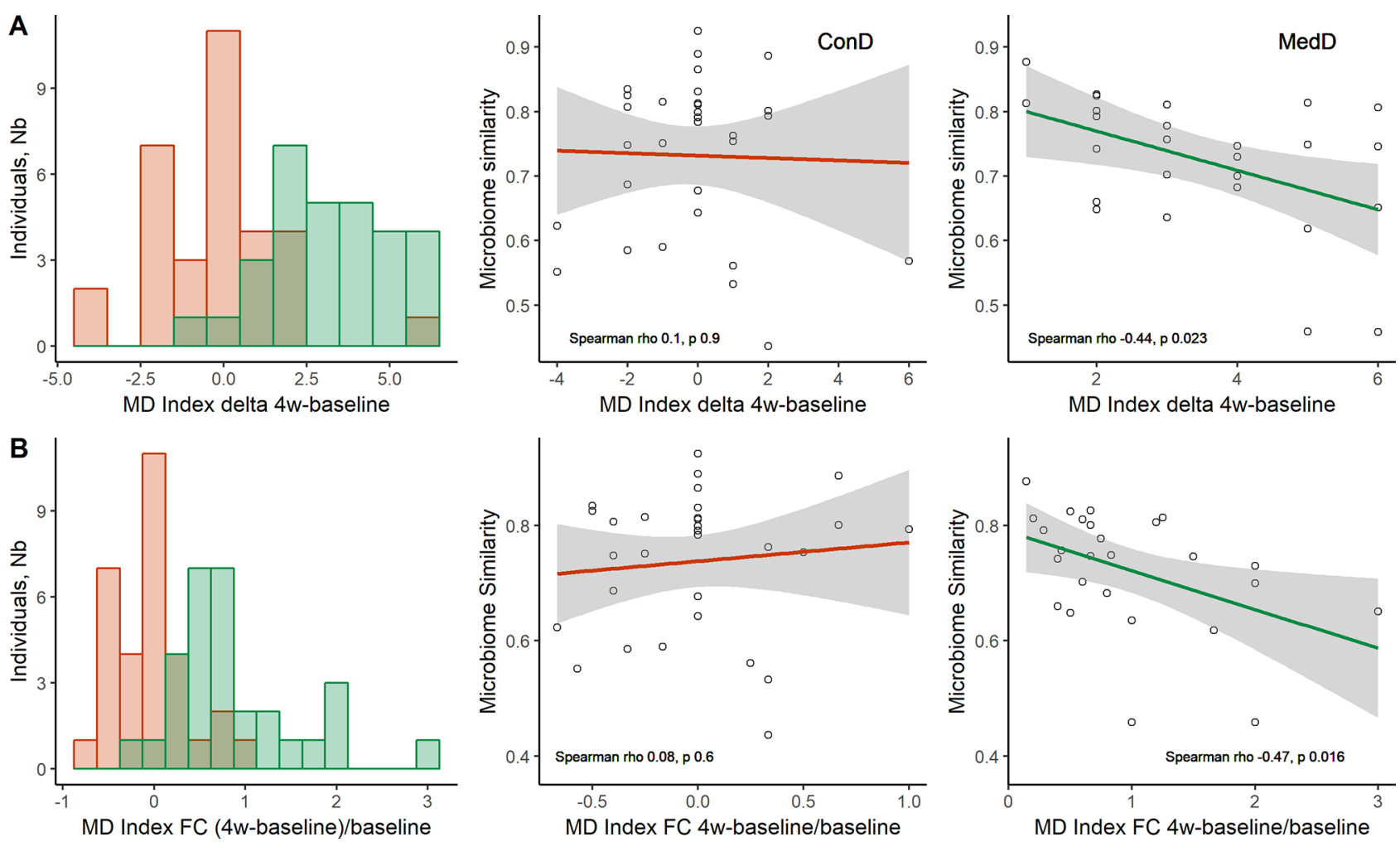

Figure 4 Mediterranean diet (MD) affects gut microbiome composition. (A) Total delta MD index changes over the 4w-baseline period. Left: histograms of delta ( $4 \mathrm{w}$-baseline) MD index ( $n=62$ ). Right: linear regressions of microbiome similarity compared with delta $4 \mathrm{w}$-baseline MD index. Microbiome similarity was estimated by Spearman correlations between microbial composition at 4 weeks and baseline within each individual. (B) Total MD index fractional changes (FCs) (4w-baseline)/baseline, used as proxy to measure the effort of adherence ( $n=62)$. Left: distribution of individuals relative to MD index FC. Right: linear regressions of microbiome similarity and FC. Spearman correlations (rho and $p$ values) are reported, excluding outliers, for ConD ( $n=31)$ and MedD ( $n=26)$ groups, respectively. 4w, 4 weeks.

was negatively correlated with serum hs-CRP, triglycerides, body fat mass, body weight, BMI and urinary carnitine (false discovery rate $(\mathrm{FDR})<0.05$, online supplementary figure 32 ).

Compared with the baseline values, a significant reduction in faecal concentrations of total BAs, including both primary and secondary BAs, was observed in the MedD group on the MD intervention (figure 5A-C). In addition, faecal deoxycholic acid was significantly reduced after $4(\mathrm{p}<0.01)$ and 8 weeks of the intervention $(p<0.01)$ along with faecal lithocholic acid $(\mathrm{p}<0.05$ and $\mathrm{p}<0.01$ after 4 and 8 weeks, respectively) within the MedD group. Paired Wilcoxon rank-sum tests of faecal BA concentrations within each intervention group are shown in online supplementary figure 33. A comparison of faecal BA concentrations between the MedD and ConD groups after 8 weeks showed a significant reduction in faecal chenodeoxycholic acid $(\mathrm{p}<0.05)$. Accordingly, primary and secondary BAs in the faeces were positively linked to proteins and fats from animal-based food products, as well as systolic blood pressure, BMI, body weight and urinary carnitine $(\mathrm{FDR}<0.05$, online supplementary figure 32).

It was also noteworthy that subjects showing the highest reduction in total BAs and the secondary/primary BA ratio had higher baseline levels of Bilophila wadsworthia, which decreased significantly after 4 weeks of the intervention $(p<0.05$, figure $5 \mathrm{D})$.

Despite the twofold increase in dietary fibre intake, no changes in faecal concentrations of the main SCFAs acetate, butyrate and propionate were observed. However, significant reductions in branched-chain fatty acids (BCFAs), such as valerate, isovalerate, isobutyrate and 2-methylbutyrate, were observed in the faeces of the participants in the MedD group over the intervention (figure 6A-D), and these changes mirrored the increased intake of plant-based foods $(\mathrm{FDR}<0.05$, online supplementary figure 32). Moreover, subjects in the quartile of the highest faecal butyrate increase at 4 weeks showed

Table 1 Urinary urolithins-glucuronides levels (ng/ $\mu$ mol creatinine) detected over the study period

\begin{tabular}{|c|c|c|c|c|c|c|c|c|}
\hline & \multicolumn{3}{|l|}{ MedD } & \multicolumn{3}{|l|}{ ConD } & \multicolumn{2}{|l|}{$P$ values } \\
\hline & Baseline & 4 & $8 w$ & Baseline & $4 w$ & $8 w$ & $\Delta_{\text {(4w-baseline) }}$ & $\Delta_{\text {(8w- baseline) }}$ \\
\hline Urolithin-A-glucuronide & $30.8 \pm 37.7$ & $139.8 \pm 296.8$ & $214.4 \pm 358$ & $5.4 \pm 30.5$ & $6.9 \pm 35.7$ & $5 \pm 28.3$ & 0.013 & 0.025 \\
\hline Urolithin-B-glucuronide & $0.1 \pm 0.7$ & $21.7 \pm 60.6$ & $74.1 \pm 243.4$ & $0.2 \pm 0.9$ & $0.1 \pm 0.5$ & $5.4 \pm 30.6$ & 0.0073 & 0.086 \\
\hline Urolithin-C-glucuronide & $1.6 \pm 8.8$ & $46.8 \pm 107.2$ & $43.2 \pm 176.6$ & $0 \pm 0$ & $0 \pm 0$ & $0 \pm 0$ & 0.021 & 0.16 \\
\hline Total urolithins $(A+B+C)$ & $32.6 \pm 91.8$ & $208.3 \pm 373.8$ & $336.7 \pm 594.3$ & $5.5 \pm 30.5$ & $7 \pm 35.7$ & $10.4 \pm 41$ & 0.00034 & 0.033 \\
\hline
\end{tabular}

Data are expressed as mean \pm SD. $P$ values refer to variation at the specific time point compared with baseline in MedD versus ConD measured by unpaired Wilcoxon rank-sum test.

$4 \mathrm{w}, 4$ weeks; $8 \mathrm{w}, 8$ weeks. 
A

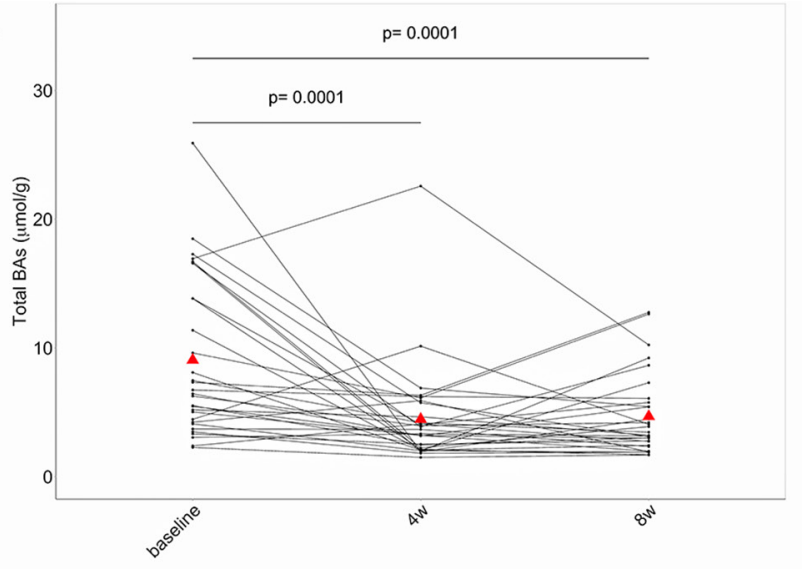

$\mathrm{C}$

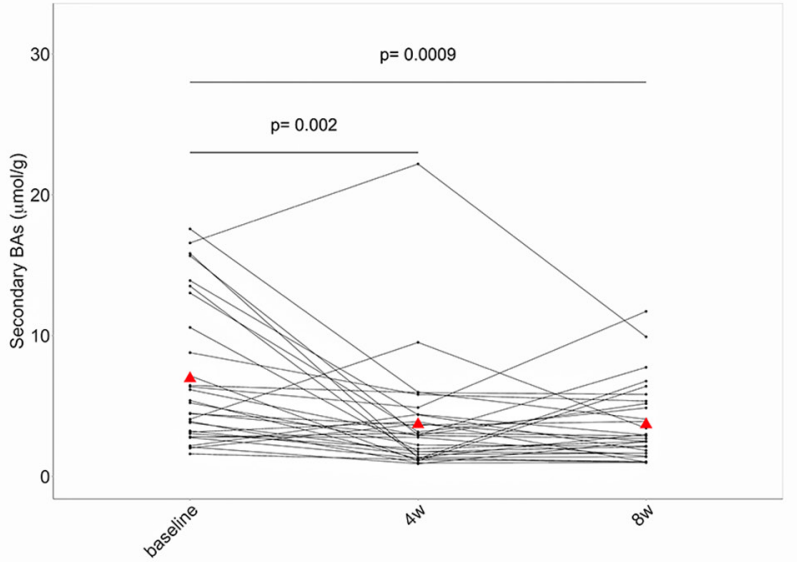

B 25

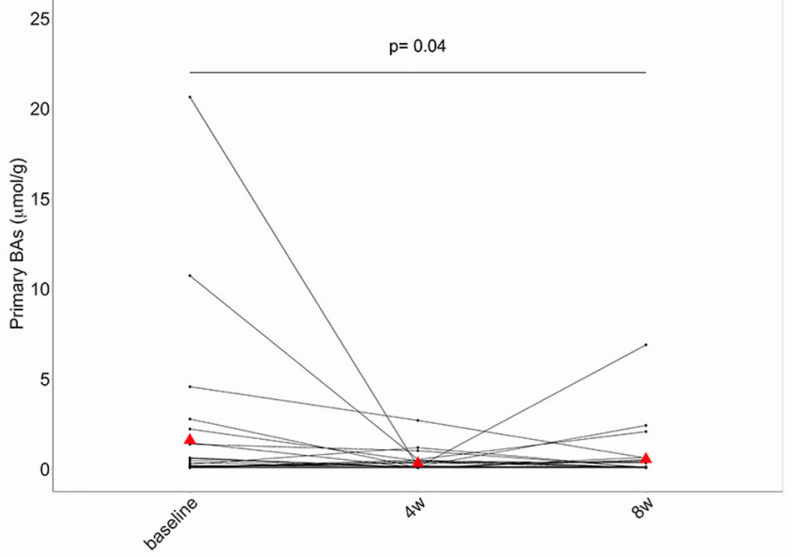

$\mathrm{D}$

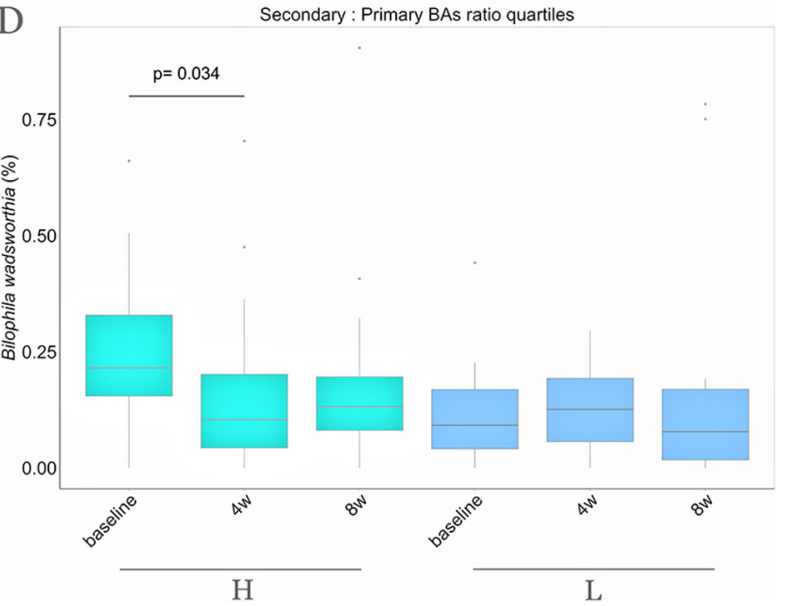

Figure 5 Faecal BA concentrations over the nutritional intervention. Parallel coordinate plot showing variations of faecal (A) total, (B) primary and (C) secondary BA concentrations within the MedD group during the intervention. The red triangles indicate mean values, the lines connecting dots are used to indicate the same sample at each time point. The significance was tested by applying the post hoc Friedman-Nemenyi test for pairwise test of multiple comparisons within each group. (D) In the box plot the relative abundances of Bilophila wadsworthia are compared considering subjects falling in the highest quartile ( $n=16$, green) and in the lowest quartile of reduction $(n=16$, blue) of secondary to primary BAs ratio after 4 weeks of treatment. Baseline, 0 weeks; 4w, 4 weeks; 8 w, 8 weeks of nutritional intervention. $H$, highest quartile of reduction; L, lowest quartile of reduction; BAs, bile acids.

consistently higher levels of $F$. prausnitzii and Lachnospiraceae taxa (figure 6E,F).

\section{Variation in insulin resistance is linked to baseline levels of specific microbial taxa}

The Homeostatic Model Assessment for Insulin Resistance (HOMA) was calculated as a measure of insulin resistance, and it did not change as a result of the intervention (online supplementary table 1). However, by stratifying the subjects by the variation in HOMA at 4 weeks compared with baseline, we found that subjects who reduced their HOMA on the MD intervention had significantly higher baseline levels of several Bacteroides species (including B. uniformis and B. vulgatus, $\mathrm{p}<0.05$ ) and lower Prevotella sp. and P. copri levels $(\mathrm{p}<0.05)$ than subjects who did not exhibit changes in HOMA over time (online supplementary figure 34). Interestingly, P. copri baseline levels showed a positive correlation with HOMA variation over the intervention (Spearman's rho $=0.28 ; \mathrm{p}=0.031$ ).

Consistently, when we computed co-abundance groups (CAGs) from $16 \mathrm{~S}$ rRNA gene sequencing analysis, we found significantly lower levels of CAG2 (including Prevotella as the most abundant genus) in subjects who exhibited reduced HOMA, while levels of CAG4 (including Faecalibacterium, Roseburia, Bacteroides, other Clostridia) were significantly higher at baseline in participants who exhibited reduced HOMA and increased on dietary treatment (online supplementary figure 35 ).

The pangenome of the HOMA and serum hs-CRP-associated species (P. copri, F. prausnitzii, B. uniformis, B. vulgatus) was further investigated. No clear differences were found according to intervention, increase in dietary fibre consumption or decrease in HOMA indicating a high subject specificity at the strain level (online supplementary figure 36 ).

\section{DISCUSSION}

This study clearly shows that a change from a Western diet to a Mediterranean dietary pattern, without any concomitant change in energy intake, macronutrient intake or physical activity, modulates individual clinical outcomes, the gut microbiome and metabolome after 4 weeks of the intervention in a population with cardiometabolic risk due to unhealthy lifestyle.

Each participant in the MedD group received a diet that was tailored to his/her habitual energy and macronutrient intake to increase the adherence to a typical MD pattern. In other words, each subject was instructed on the exact replacements of foods 

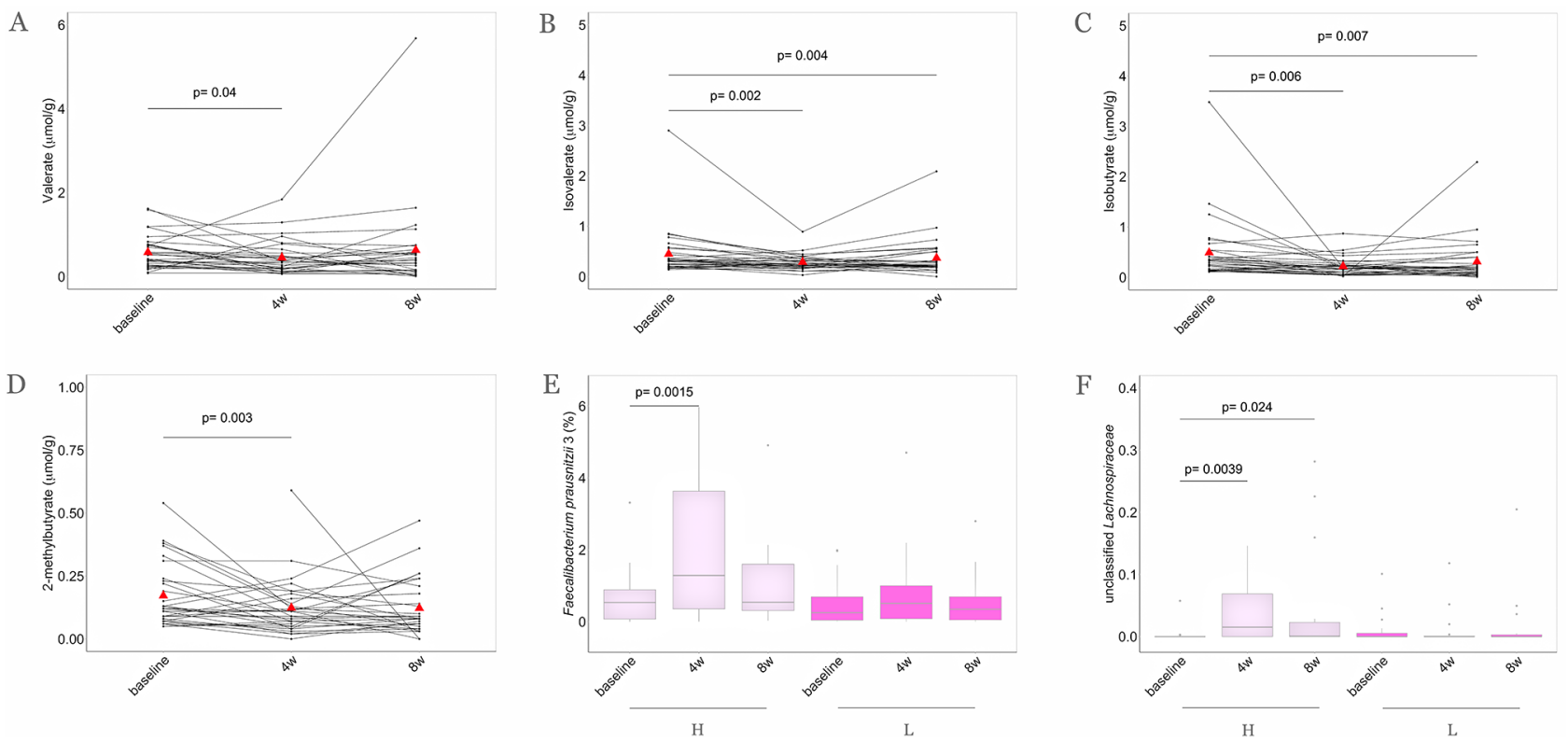

Figure 6 MD intervention determines a reduction of faecal branched-chain fatty acid (BCFA) concentrations and higher levels of Faecalibacterium prausnitzii and Lachnospiraceae taxa. Parallel coordinate plot showing variations of (A) valerate, (B) isovalerate, (C) isobutyrate and (D) 2-methylbutyrate faecal concentrations within MedD population. The red triangles indicate mean values, the lines connecting dots are used to indicate the same sample at each time point. The significance was tested by applying the post hoc Friedman-Nemenyi test for pairwise test of multiple comparisons within each group. In the box plots, the relative abundances of (E) F. prausnitzii 3 and (F) Lachnospiraceae family are compared considering subjects falling in the highest quartile $(n=16$, violet) and in the lowest quartile $(n=16$, purple) of faecal butyrate increase after 4 weeks of treatment. Statistical differences between groups were determined using Wilcoxon rank-sum tests. Baseline, 0 weeks; $4 \mathrm{w}$, 4 weeks; $8 \mathrm{w}$, 8 weeks of intervention. $\mathrm{H}$, highest quartile of increase; $\mathrm{L}$, lowest quartile of increase.

so that specific amounts of Western diet foods were replaced by the ones typical of an MD. At the best of our knowledge, this approach has not been previously used in intervention studies with the MD. This ensured that changes in metabolic markers, the gut microbiome and systemic metabolome were not biassed by variation in energy intake over the nutritional intervention.

From a clinical perspective, the data show that within a short period, consumption of an MD can lower total, LDL-cholesterol and HDL-cholesterol in plasma independently of individual energy intake and physical activity level. The decrease in LDLcholesterol (by $0.24 \mathrm{mmol} / \mathrm{L},-8.3 \%$ vs baseline) associated with MD in this study is far from the reduction of $1 \mathrm{mmol} / \mathrm{L}$ that has been indicated as clinically relevant towards a reduction in heart disease risk. ${ }^{35}$ However, it is higher than the average reductions (between 0.11 and $0.23 \mathrm{mmol} / \mathrm{L}$ ) that have been found in RCTs comparing meat-based diets with plant-proteinbased diets including nuts or legumes separately, ${ }^{36}$ as well as those achievable $(\sim 0.1 \mathrm{mmol} / \mathrm{L})$ with diets including ellagitannins or anthocyanins. ${ }^{37}$ Interestingly, in the current study, the cholesterol-lowering effect was linearly associated with individual adherence to the MD. The relationship between the MD index and plasma cholesterol highlights the importance of the whole MD pattern and of individual dietary compliance in eliciting the hypolipidaemic effect of the MD. We hypothesise that the lower dietary intakes of cholesterol $(p<0.0001$ at 4 and 8 weeks vs baseline) and saturated fats $(\mathrm{p}=0.005$ at 4 and at 8 weeks vs baseline) on the MD intervention are the main factors responsible for that effect. ${ }^{38}$

Adherence to the MD was confirmed by comprehensive untargeted metabolic profiling of faeces, serum and urine, as well as targeted quantification of selected biomarkers. In agreement with the MD pattern, we found increased levels of biomarkers of wholegrains, legumes, vegetables and nuts, as well as reduced concentrations of biomarkers of meat and protein degradation products after the MD intervention. These objective measures substantiated the dietary records obtained by the Food Frequency Questionnaires (FFQ) and the 7-day food diary. The MD-dependent metabolome shift was particularly evident in the urine metabolome due to the accumulation of diet-derived metabolites of wholegrains, nuts and vegetables. In addition, a range of shortchain and medium-chain acylcarnitines were consistently reduced in urine following the MD intervention, suggesting a diet-induced shift in energy production from beta-oxidation to glycolysis in the mitochondria, probably due to an extended period of carbohydrate availability due to a steady release from fibre degradation. In agreement with these findings, plasma short-chain acylcarnitines have been associated with a Western $\operatorname{diet}^{39}$ and have been found in higher concentrations in meat eaters than in vegetarians and vegans, ${ }^{40}$ and urine levels of acylcarnitines were reduced with increased wholegrain intake. ${ }^{41}$ Since acylcarnitines have been associated with an increased risk of $\mathrm{CVD},{ }^{42}$ the reduction in acylcarnitines in urine suggests a beneficial MD-induced effect on energy metabolism caused by increased intake of dietary fibre.

Overall, the differences in the faecal metabolome associated with the intervention reflect the replacement of foods of animal origin with plant-based foods following MD adherence. Oxindole-3-acetic acid, a naturally occurring auxin in plants, ${ }^{43}$ as well as the BCAAs leucine and isoleucine and BAs appeared to be the main drivers. BAs can be implicated in atherosclerosis, diabetes and other cardiometabolic diseases. ${ }^{44}$ Targeted quantification of faecal BAs confirmed a significant reduction in their concentrations within the MedD group coherently with the reduced intake of meat products. In line with these findings, a vegan diet has been found to reduce plasma BCAAs and BAs in comparison with the levels associated with an animal-based diet. $^{45}$ 
High adherence to an MD has also been associated with increased faecal concentrations of SCFAs. ${ }^{46}$ Despite the fact that participants doubled their intake of dietary fibre, the MD intervention did not significantly increase the faecal concentrations of SCFAs. Faecal SCFA represents the difference between the production and absorption or utilisation of SCFAs in the colon and rectum. We speculate that a possible improved gut epithelial function may have increased SCFAs utilisation and absorption, thus hampering the observation of their increase due to higher fibre intake. This result was corroborated by recent findings that dysbiosis is associated with increased faecal SCFA excretion. ${ }^{47}$ The MD decreased faecal concentrations of BCFAs, including valerate, which is in agreement with previous studies reporting faecal valerate as linked to the consumption of protein-rich animal foods ${ }^{46}$ and not to MD adherence. ${ }^{48}$ These results suggest an altered colonic proteolytic fermentation caused by the replacement of animal-based products with plant-based foods. This finding was substantiated by microbial-derived proteolytic products being reduced with increased MD adherence. An interesting increase in urolithins was observed in the MedD group.

Urolithins are gut microbial metabolites of ellagitannins. ${ }^{49}$ Dietary sources of these polyphenols are berries, pomegranate and walnuts. However, our data indicated that only nut consumption significantly increased over the intervention with MD. Therefore, increase in urinary urolithin glucuronides was most likely attributed to the intake of walnuts in our study as previously reported by others. ${ }^{50}$ Recently, urolithin A has been shown to improve intestinal barrier function in a preclinical mode $^{51}$ and has also been associated with lower cardiometabolic risk. ${ }^{52}$ In addition, urolithin $\mathrm{A}$ has been demonstrated to be involved in the prevention of prostate, ${ }^{53}$ endometrial $^{54}$ and breast cancer ${ }^{55}$ in vitro. Interestingly, in our intervention study, urolithin levels were negatively correlated with cardiometabolic risk factors such as triglycerides and BMI and these observations further corroborate the hypothesis that an MD dietary pattern might beneficially impacts human health status through gut microbiota metabolism.

It was recently reported that microbiome composition is more associated with specific food choices than with nutritional patterns, that food-microbe interactions are highly personalised, and that these factors might limit the observation of overall microbiome responses to specific diets. ${ }^{9}$ Interestingly, despite such insightful evidence, we observed clear microbiome shifts following our dietary intervention protocol.

Gut microbial taxonomic and functional composition in our isocaloric MD intervention revealed that the overall microbial richness was maintained, which is consistent with recent studies showing similar trends after increased consumption of wholegrain. ${ }^{2856}$ However, we observed that the MD dynamically modulates the intestinal microbiome composition and that the microbiome variations are proportional to the increase in MD adherence rates.

Even though prior studies addressed the link between diet, gene richness and inflammation markers, ${ }^{72856-58}$ intervention studies describing variation of the microbial genetic richness following an MD dietary pattern have not previously been described. Interestingly, here MD improves the inflammatory status of individuals experiencing an increase in gut microbiome gene richness during controlled energy and modified macronutrient intakes, further supporting the idea that MD might be an efficient dietary strategy to reduce inflammation. ${ }^{1359}$

The MD intervention protocol determined a decline in $R$. torques and $R$. gnavus. The latter species has been recently demonstrated as a proinflammatory species due to secretion of a polysaccharide that induces tumour necrosis factor alpha in dendritic cells, ${ }^{60}$ whereas possible involvement of $R$. torques in inflammation remains largely uncertain and is currently based on associations. ${ }^{6162}$

Subjects with the highest reduction in faecal BAs consistently also exhibited reduced relative abundance of Bilophila wadsworthia, which was previously linked to higher BA levels, ${ }^{63}$ animalbased and high fat diets, as well as irritable bowel diseases. ${ }^{764}$ This decline was accompanied by an increase in several potentially beneficial species, including the fibre-degrading F. prausnitzii, Roseburia and members of the Clostridiales and Lachnospiraceae taxa, linked to butyrate precursor functional pathways. These reportedly beneficial species were previously documented for their anti-inflammatory properties and their role in the development of the intestinal barrier ${ }^{656}$ and were, in the present study, found to be boosted by foods recommended as part of a healthy MD nutritional pattern.

Our data also show that an MD-tailored dietary intervention might be helpful in ameliorating insulin sensitivity in individuals harbouring higher levels of several Bacteroides species and lower levels of Prevotella sp. and P. copri. The association of $P$. copri with insulin resistance was already reported by Pedersen et $a^{67}$ and it was recently demonstrated to be strain-dependent and correlated with the occurrence of genes involved in BCAA biosynthesis. ${ }^{68}$

These findings are in line with the concept of personalised responses of individuals to similar diets, and they are of importance for clinical practice in the era of precision medicine and personalised nutrition. ${ }^{912}$

Taken together, our results indicate that an MD may remodel the intestinal microbiome towards a state that promotes metabolic and cardiovascular health. In addition, our observations can be useful to plan baseline stratifications of subjects based on microbiome composition to select specific metabotypes that could be involved in ad hoc nutritional interventions to potentiate the clinical outcomes.

\section{Author affiliations}

${ }^{1}$ Université Paris-Saclay, INRAE, MGP, Jouy-en-Josas, France

${ }^{2}$ Department of Agricultural Sciences, University of Naples Federico II, Portici, Italy ${ }^{3}$ Department of Nutrition, Exercise and Sports, Faculty of Science, University of Copenhagen, Frederiksberg, Denmark

${ }^{4}$ Task Force on Microbiome Studies, University of Naples Federico II, Naples, Italy ${ }^{5}$ Institute of Food Science of CNR, Avellino, Italy

${ }^{6}$ Department of Clinical Medicine and Surgery, University of Naples Federico II, Naples, Italy

Twitter Manolo Laiola @hroager, Henrik Munch Roager @hroager and Danilo Ercolini @DaniloErcolini

Acknowledgements We would like to thank Agria Spa, Fiammante Icab Spa, Olio Dante Spa and Pastificio Di Martino Spa for kindly providing foods to be included in the food boxes for our participants. The authors would also like to thank Cecilie Fryland Appeldorff, Sofie Skov Frost and Sarah Fleischer Ben Soltane (University of Copenhagen) for excellent technical assistance on ultra-high-performance liquid chromatography mass spectrometry (UHPLC-MS) analyses; Sébastien Fromentin, Magali Berland, Kévin Da Silva and Florence Thirion (MGP Metagenopolis) for their distinguished bioinformatical guidance; and Dr Maria Assunta Gallo and Centro Diagnostico San Ciro (Portici, Italy) for their support with the clinical visits.

Contributors The principal investigators (DE, DSE and LOD) and the other coapplicants (PV) designed the study and obtained funding. The nutritional intervention was carried out at the University of Naples Federico II by AAR, RG, IM and PV. HMR, RF, LOD and PV generated and analysed the metabolomics data. HR and $B Q$ generated the metagenomics data, and ML, VM, FDF, EP and NP analysed the microbiome data and performed the statistical analyses. All authors participated in the data interpretation; DE wrote the paper with contributions by ML, HMR, PV and VM. All authors read, edited and approved the final version of the manuscript. The corresponding authors attest that all listed authors meet authorship criteria and that no others meeting the criteria have been omitted. 
Funding The study was conducted within the Diet-Induced Arrangement of the gut Microbiome for the Improvement of Cardiometabolic health (DINAMIC) project funded within the European Joint Programming Initiative "A Healthy Diet for a Healthy Life" (JPI HDHL) - Joint Action "Intestinal Microbiomics". The DINAMIC national funding organization are (i) the Italian Ministry of Education, University and Research (prot.0000426); (ii) the Innovation Fund Denmark (grant \# 5195-00001B) and (iii) the French National Research agency (ANR-15-HDIM-0002-04). The study was also partially supported by a Semper Ardens grant to Lars Ove Dragsted and the Metagenopolis grant ANR-11-DPBS-0001.A full list of the DINAMIC consortium and their affiliations appears in the online supplementary material.

Competing interests None declared.

Patient consent for publication Not required.

Ethics approval The trial was conducted at the University of Naples Federico II and was approved by the related Ethics Committee (Protocol number: 108/16)

Provenance and peer review Not commissioned; externally peer reviewed.

Data availability statement Data are available in a public, open access repository. Metagenomic reads generated in this study are available (without conditions of reuse) under the accession number PRJEB33500 at the European Nucleotide Archive (ENA) in EBI (https://www.ebi.ac.uk/ena/data/view/PRJEB33500).

Open access This is an open access article distributed in accordance with the Creative Commons Attribution Non Commercial (CC BY-NC 4.0) license, which permits others to distribute, remix, adapt, build upon this work non-commercially, and license their derivative works on different terms, provided the original work is properly cited, appropriate credit is given, any changes made indicated, and the use is non-commercial. See: http://creativecommons.org/licenses/by-nc/4.0/.

\section{ORCID iDs}

Henrik Munch Roager http://orcid.org/0000-0002-2504-8313

Lars Ove Dragsted http://orcid.org/0000-0003-0609-6317

Stanislav Dusko Ehrlich http://orcid.org/0000-0002-7563-4046

Danilo Ercolini http://orcid.org/0000-0003-3061-9560

\section{REFERENCES}

1 Kelly JT, Palmer SC, Wai SN, et al. Healthy dietary patterns and risk of mortality and ESRD in CKD: a meta-analysis of cohort studies. Clin J Am Soc Nephrol 2017;12:272-9.

2 Chiavaroli L, Viguiliouk E, Nishi SK, et al. Dash dietary pattern and cardiometabolic outcomes: an umbrella review of systematic reviews and meta-analyses. Nutrients 2019;11:338

3 Martínez-González MA, Gea A, Ruiz-Canela M. The Mediterranean diet and cardiovascular health. Circ Res 2019;124:779-98.

4 Bendall CL, Mayr HL, Opie RS, et al. Central obesity and the Mediterranean diet: a systematic review of intervention trials. Crit Rev Food Sci Nutr 2018;58:3070-84.

5 Eleftheriou D, Benetou V, Trichopoulou A, et al. Mediterranean diet and its components in relation to all-cause mortality: meta-analysis. Br J Nutr 2018;120:1081-97.

6 Cani PD. Human gut microbiome: hopes, threats and promises. Gut 2018;67:1716-25

7 David LA, Maurice CF, Carmody RN, et al. Diet rapidly and reproducibly alters the human gut microbiome. Nature 2014;505:559-63.

8 Roager HM, Dragsted LO. Diet-derived microbial metabolites in health and disease. Nutr Bull 2019:44:216-27.

9 Johnson AJ, Vangay P, Al-Ghalith GA, et al. Daily sampling reveals personalized DietMicrobiome associations in humans. Cell Host Microbe 2019;25:789-802.

10 Bashiardes S, Godneva A, Elinav E, et al. Towards utilization of the human genome and microbiome for personalized nutrition. Curr Opin Biotechnol 2018:51:57-63.

11 Grosso G, Micek A, Godos J, et al. Health risk factors associated with meat, fruit and vegetable consumption in cohort studies: a comprehensive meta-analysis. PLoS One 2017; 12:e0183787.

12 De Filippis F, Vitaglione P, Cuomo R, et al. Dietary interventions to modulate the gut Microbiome-How far away are we from precision medicine. Inflamm Bowel Dis 2018:24:2142-54

13 Bailey MA, Holscher HD. Microbiome-mediated effects of the Mediterranean diet on inflammation. Adv Nutr 2018;9:193-206

14 Harris RP, Helfand M, Woolf $\mathrm{SH}$, et al. Current methods of the US preventive services Task force: a review of the process. Am J Prev Med 2001;20:21-35.

15 Blumberg J, Heaney RP, Huncharek M, et al. Evidence-Based criteria in the nutritiona context. Nutr Rev 2010;68:478-84.

16 Myles IA. Fast food fever: reviewing the impacts of the Western diet on immunity. Nutr J 2014;13:61

17 Minihane AM, Vinoy S, Russell WR, et al. Low-Grade inflammation, diet composition and health: current research evidence and its translation. Br J Nutr 2015;114:999-1012

18 Mozaffarian D, Hao T, Rimm EB, et al. Changes in diet and lifestyle and long-term weight gain in women and men. N Engl J Med 2011;364:2392-404
19 Agnoli C, Krogh V, Grioni S, et al. A priori-defined dietary patterns are associated with reduced risk of stroke in a large Italian cohort. J Nutr 2011;141:1552-8.

20 Andersen M-BS, Kristensen M, Manach C, et al. Discovery and validation of urinary exposure markers for different plant foods by untargeted metabolomics. Anal Bioanal Chem 2014;406:1829-44

21 Barri T, Dragsted LO. UPLC-ESI-QTOF/MS and multivariate data analysis for blood plasma and serum metabolomics: effect of experimental artefacts and anticoagulant. Anal Chim Acta 2013;768:118-28.

22 Christiansen CB, Gabe MBN, Svendsen B, et al. The impact of short-chain fatty acids on GLP-1 and PYY secretion from the isolated perfused rat colon. Am J Physiol Gastrointest Liver Physiol 2018;315:G53-65.

23 Hjerpsted JB, Dragsted LO, Tholstrup T. Cheese intake lowers plasma cholesterol concentrations without increasing bile acid excretion. J Nutr Intermed Metab 2016;3:12-17.

24 Plaza Oñate F, Le Chatelier E, Almeida M, et al. MSPminer: abundance-based reconstitution of microbial pan-genomes from shotgun metagenomic data. Bioinformatics 2019:35:1544-52.

25 Gu Z, Eils R, Schlesner M. Complex heatmaps reveal patterns and correlations in multidimensional genomic data. Bioinformatics 2016:32:2847-9.

26 Pasolli E, Truong DT, Malik F, et al. Machine learning meta-analysis of large metagenomic datasets: tools and biological insights. PLoS Comput Biol 2016;12:e1004977

27 Drescher LS, Thiele S, Mensink GBM. A new index to measure healthy food diversity better reflects a healthy diet than traditional measures. J Nutr 2007;137:647-51.

28 Roager HM, Vogt JK, Kristensen M, et al. Whole grain-rich diet reduces body weight and systemic low-grade inflammation without inducing major changes of the gut microbiome: a randomised cross-over trial. Gut 2019:68:83-93.

29 Keller BO, Wu BTF, Li SSJ, et al. Hypaphorine is present in human milk in association with consumption of legumes. J Agric Food Chem 2013;61:7654-60.

30 Cuparencu CS, Andersen M-BS, Gürdeniz G, et al. Identification of urinary biomarkers after consumption of sea buckthorn and strawberry, by untargeted LC-MS metabolomics: a meal study in adult men. Metabolomics 2016;12:31

31 Tulipani S, Urpi-Sarda M, García-Villalba R, et al. Urolithins are the main urinary microbial-derived phenolic metabolites discriminating a moderate consumption of nuts in free-living subjects with diagnosed metabolic syndrome. J Agric Food Chem 2012;60:8930-40

32 Dragsted LO. Biomarkers of meat intake and the application of nutrigenomics. Meat Sci 2010:84:301-7.

33 Khakimov B, Poulsen SK, Savorani F, et al. New Nordic diet versus average Danish diet: a randomized controlled trial revealed healthy long-term effects of the new Nordic diet by GC-MS blood plasma metabolomics. J Proteome Res 2016:15:1939-54

34 Singh A, Shannon CP, Gautier B, et al. DIABLO: an integrative approach for identifying key molecular drivers from multi-omics assays. Bioinformatics 2019;35:3055-62.

35 Lewington S, Whitlock G, Clarke R, et al. Blood cholesterol and vascular mortality by age, sex, and blood pressure: a meta-analysis of individual data from 61 prospective studies with 55,000 vascular deaths. Lancet 2007;370:1829-39.

36 Guasch-Ferré M, Satija A, Blondin SA, et al. Meta-Analysis of randomized controlled trials of red meat consumption in comparison with various comparison diets on cardiovascular risk factors. Circulation 2019;139:1828-45.

37 García-Conesa M-T, Chambers K, Combet E, et al. Meta-Analysis of the effects of foods and derived products containing ellagitannins and anthocyanins on cardiometabolic biomarkers: analysis of factors influencing variability of the individual responses. Int J Mol Sci 2018:19:694

38 Wolff $E$, Vergnes M-F, Portugal $H$, et al. Cholesterol-absorber status modifies the LDL cholesterol-lowering effect of a Mediterranean-type diet in adults with moderate cardiovascular risk factors. J Nutr 2011;141:1791-8.

39 Bouchard-Mercier A, Rudkowska I, Lemieux S, et al. The metabolic signature associated with the Western dietary pattern: a cross-sectional study. Nutr J 2013;12:158.

40 Schmidt JA, Rinaldi S, Ferrari P, et al. Metabolic profiles of male meat eaters, fish eaters, vegetarians, and vegans from the EPIC-Oxford cohort. Am J Clin Nutr 2015:102:1518-26.

41 Ross AB, Pere-Trépat E, Montoliu I, et al. A whole-grain-rich diet reduces urinary excretion of markers of protein catabolism and gut microbiota metabolism in healthy men after one week. J Nutr 2013;143:766-73.

42 Guasch-Ferré $M$, Zheng Y, Ruiz-Canela $M$, et al. Plasma acylcarnitines and risk of cardiovascular disease: effect of Mediterranean diet interventions. Am J Clin Nutr 2016;103:1408-16.

43 Korver RA, Koevoets IT, Testerink C. Out of shape during stress: a key role for auxin. Trends Plant Sci 2018;23:783-93.

44 Chávez-Talavera 0, Tailleux A, Lefebvre P, et al. Bile acid control of metabolism and inflammation in obesity, type 2 diabetes, dyslipidemia, and nonalcoholic fatty liver disease. Gastroenterology 2017:152:1679-94.

45 Draper CF, Vassallo I, Di Cara A, et al. A 48-hour vegan diet challenge in healthy women and men induces a branch-chain amino acid related, health associated, metabolic signature. Mol Nutr Food Res 2018;62:1700703. 
46 De Filippis F, Pellegrini N, Vannini L, et al. High-Level adherence to a Mediterranean diet beneficially impacts the gut microbiota and associated metabolome. Gut 2016;65:1812-21.

47 de la Cuesta-Zuluaga J, Mueller N, Álvarez-Quintero R, et al. Higher fecal short-chain fatty acid levels are associated with gut microbiome dysbiosis, obesity, hypertension and cardiometabolic disease risk factors. Nutrients 2019;11:51.

48 Mitsou EK, Kakali A, Antonopoulou S, et al. Adherence to the Mediterranean diet is associated with the gut microbiota pattern and gastrointestinal characteristics in an adult population. Br J Nutr 2017;117:1645-55.

49 Cerdá B, Periago P, Espín JC, et al. Identification of urolithin a as a metabolite produced by human colon microflora from ellagic acid and related compounds. J Agric Food Chem 2005;53:5571-6.

50 Garcia-Aloy M, Hulshof PJM, Estruel-Amades S, et al. Biomarkers of food intake for nuts and vegetable oils: an extensive literature search. Genes Nutr 2019;14:7.

51 Singh R, Chandrashekharappa S, Bodduluri SR, et al. Enhancement of the gut barrier integrity by a microbial metabolite through the Nrf2 pathway. Nat Commun 2019;10:89.

52 Selma MV, González-Sarrías A, Salas-Salvadó J, et al. The gut microbiota metabolism of pomegranate or walnut ellagitannins yields two urolithinmetabotypes that correlate with cardiometabolic risk biomarkers: comparison between normoweight, overweight-obesity and metabolic syndrome. Clin Nutr 2018;37:897-905.

53 Stanisławska IJ, Granica S, Piwowarski JP, et al. The activity of urolithin A and M4 Valerolactone, colonic microbiota metabolites of polyphenols, in a prostate cancer in vitro model. Planta Med 2019;85:118-25.

54 Zhang W, Chen J-H, Aguilera-Barrantes I, et al. Urolithin a suppresses the proliferation of endometrial cancer cells by mediating estrogen receptor- $\alpha$-dependent gene expression. Mol Nutr Food Res 2016;60:2387-95.

55 Teixeira LL, Costa GR, Dörr FA, et al. Potential antiproliferative activity of polyphenol metabolites against human breast cancer cells and their urine excretion pattern in healthy subjects following acute intake of a polyphenol-rich juice of grumixama (Eugenia brasiliensis Lam.). Food Funct 2017;8:2266-74.
56 Haro C, Montes-Borrego M, Rangel-Zúñiga OA, et al. Two healthy diets modulate gut microbial community improving insulin sensitivity in a human obese population. J Clin Endocrinol Metab 2016;101:233-42.

57 Haro C, Garcia-Carpintero S, Alcala-Diaz JF, et al. The gut microbial community in metabolic syndrome patients is modified by diet. J Nutr Biochem 2016;27:27-31.

58 Vanegas SM, Meydani M, Barnett JB, et al. Substituting whole grains for refined grains in a 6-wk randomized trial has a modest effect on gut microbiota and immune and inflammatory markers of healthy adults. Am J Clin Nutr 2017;105:635-50.

59 Vitaglione $\mathrm{P}$, Mennella I, Ferracane R, et al. Whole-grain wheat consumption reduces inflammation in a randomized controlled trial on overweight and obese subjects with unhealthy dietary and lifestyle behaviors: role of polyphenols bound to cereal dietary fiber. Am J Clin Nutr 2015;101:251-61.

60 Henke MT, Kenny DJ, Cassilly CD, et al. Ruminococcus gnavus, a member of the human gut microbiome associated with Crohn's disease, produces an inflammatory polysaccharide. Proc Natl Acad Sci U S A 2019;116:12672-7.

61 Le Chatelier E, Nielsen T, Qin J, et al. Richness of human gut microbiome correlates with metabolic markers. Nature 2013;500:541-6.

62 Brahe LK, Le Chatelier E, Prifti E, et al. Specific gut microbiota features and metabolic markers in postmenopausal women with obesity. Nutr Diabetes 2015;5:e159.

63 Devkota S, Wang Y, Musch MW, et al. Dietary-fat-induced taurocholic acid promotes pathobiont expansion and colitis in IL10-/- mice. Nature 2012;487:104-8.

64 Natividad JM, Lamas B, Pham HP, et al. Bilophila wadsworthia aggravates high fat diet induced metabolic dysfunctions in mice. Nat Commun 2018;9:2802.

65 Sokol H, Pigneur B, Watterlot L, et al. Faecalibacterium prausnitzii is an antiinflammatory commensal bacterium identified by gut microbiota analysis of Crohn disease patients. Proc Natl Acad Sci U S A 2008;105:16731-6.

66 Vital M, Karch A, Pieper DH. Colonic butyrate-producing communities in humans: an overview using omics data. mSystems 2017;2:e00130-17.

67 Pedersen HK, Gudmundsdottir V, Nielsen HB, et al. Human gut microbes impact host serum metabolome and insulin sensitivity. Nature 2016;535:376-81.

68 De Filippis F, Pasolli E, Tett A, et al. Distinct genetic and functional traits of human intestinal Prevotella copri strains are associated with different habitual diets. Cell Host Microbe 2019;25:444-53. 